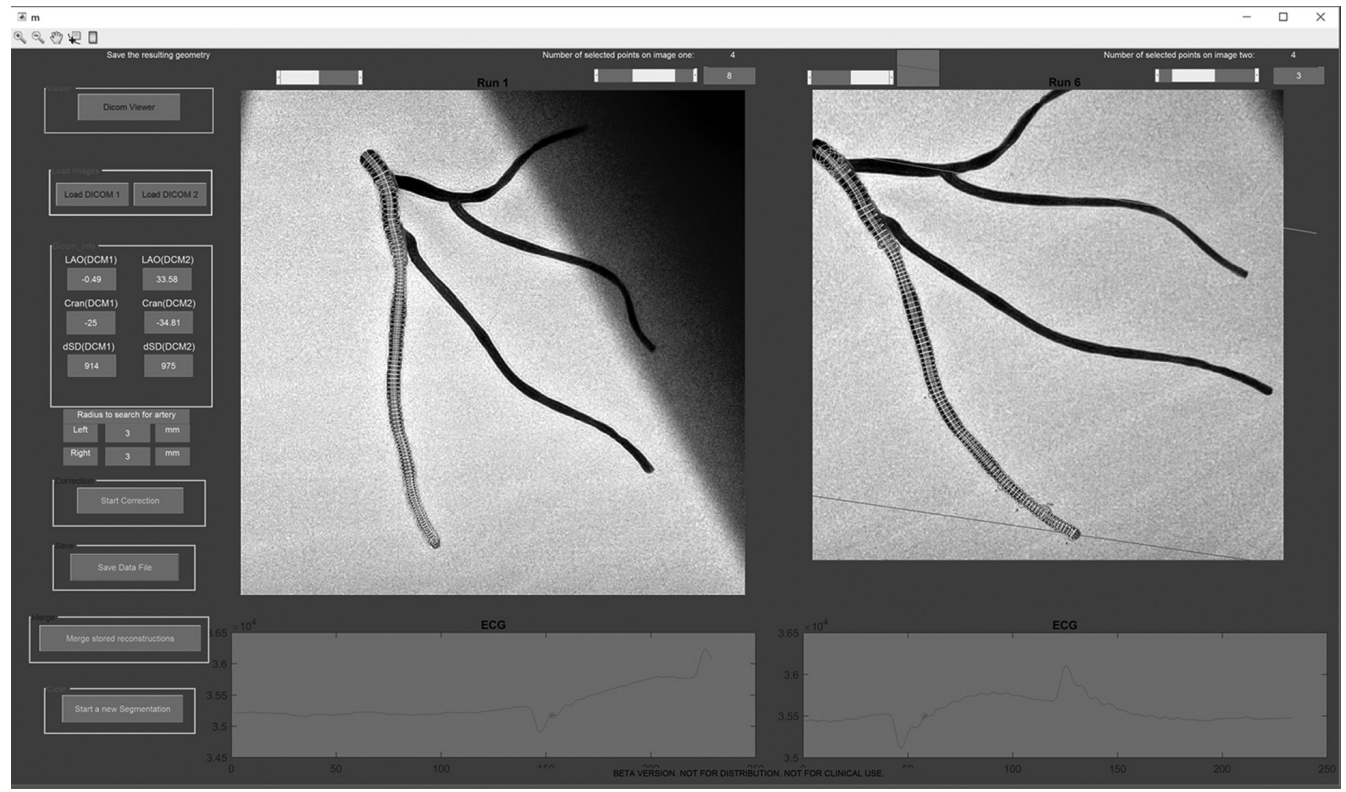

Abstract 16 Figure 1vessel reconstruction using the developed method on a left coronary model; highlighting the chosen vessel (left anterior descending artery), its centreline (yellow line) and sampled diameter measurements (yellow circles). The first DICOM image (a) shows the left anterior descending artery in good view; (b) shows a second view of the vessel $>30^{\circ}$ apart from the first.

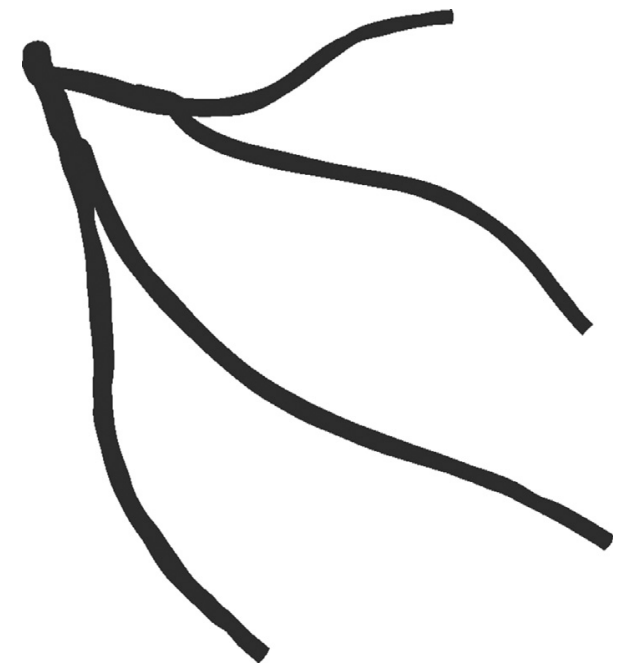

Abstract 16 Figure 2 This demonstrates the resultant 3D reconstructed left coronary system that is generated after the steps described in Figure 2. This model consists of four individual branches aligned to form a full 3D model of a left coronary system

\section{7 \\ COMPUTATIONAL MODELLING OF FRACTIONAL FLOW RESERVE FROM CORONARY ANGIOGRAPHY: EXPERT TRAINING REQUIRED}

${ }^{1}$ Katherine Lal ${ }^{*},{ }^{2}$ Julian Gunn, ${ }^{2}$ Paul Morris, ${ }^{2}$ Rebecca Gosling, ${ }^{2}$ Patricia Lawford,

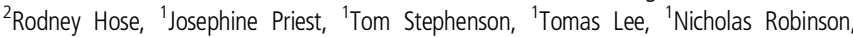
${ }^{1}$ Timothy O'Connor, 'Benjamin Gregory, 'Sein Son, ${ }^{1}$ Andrew Hodgson, ${ }^{1}$ James Dunnill. ${ }^{1}$ Department of Infection, Immunity and Cardiovascular Disease, University of Sheffield; ${ }^{2}$ University of Sheffield
Lal K, Gosling R, Priest J, Stephenson T, Lee T, Robinson N, O'Connor T, Gregory B, Son S, Hodgson A, Dunnill J, Lawford P, Hose R, Morris PD, Gunn J

Sheffield Teaching Hospitals NHS Foundation Trust and Department of Infection, Immunity and Cardiovascular Disease, University of Sheffield

Introduction Visual estimation of the physiological significance of coronary artery disease (CAD) is inaccurate. Fractional flow reserve (FFR) is better but is under-used. A less invasive alternative is 'virtual' FFR (vFFR) calculated from computational fluid dynamics (CFD) modelling from angiographic images. The aim of this study was to quality assess the vFFRs analysed by non-expert operators by comparing their results to those of fully trained experts.

Methods Two expert operators re-processed vFFRs from patients with CAD that had previously been processed by
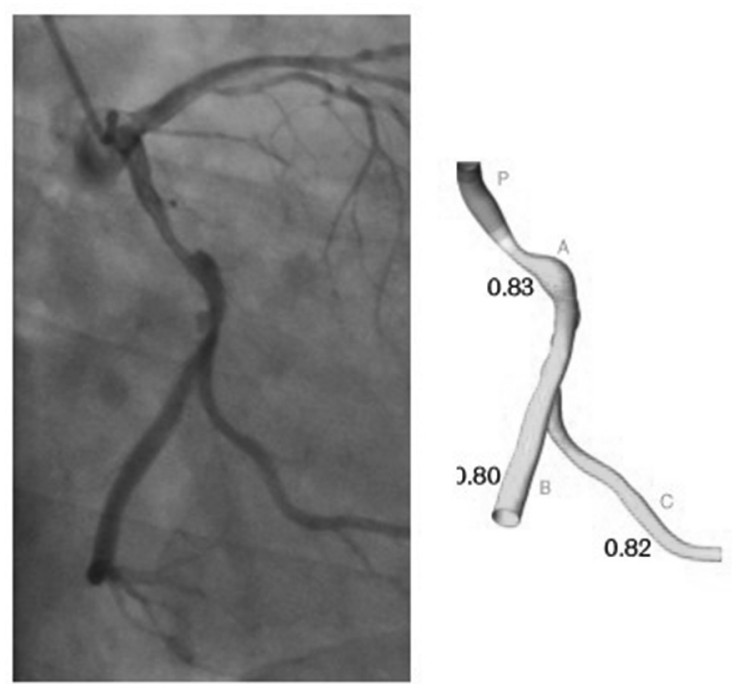

Abstract 17 Figure 1 


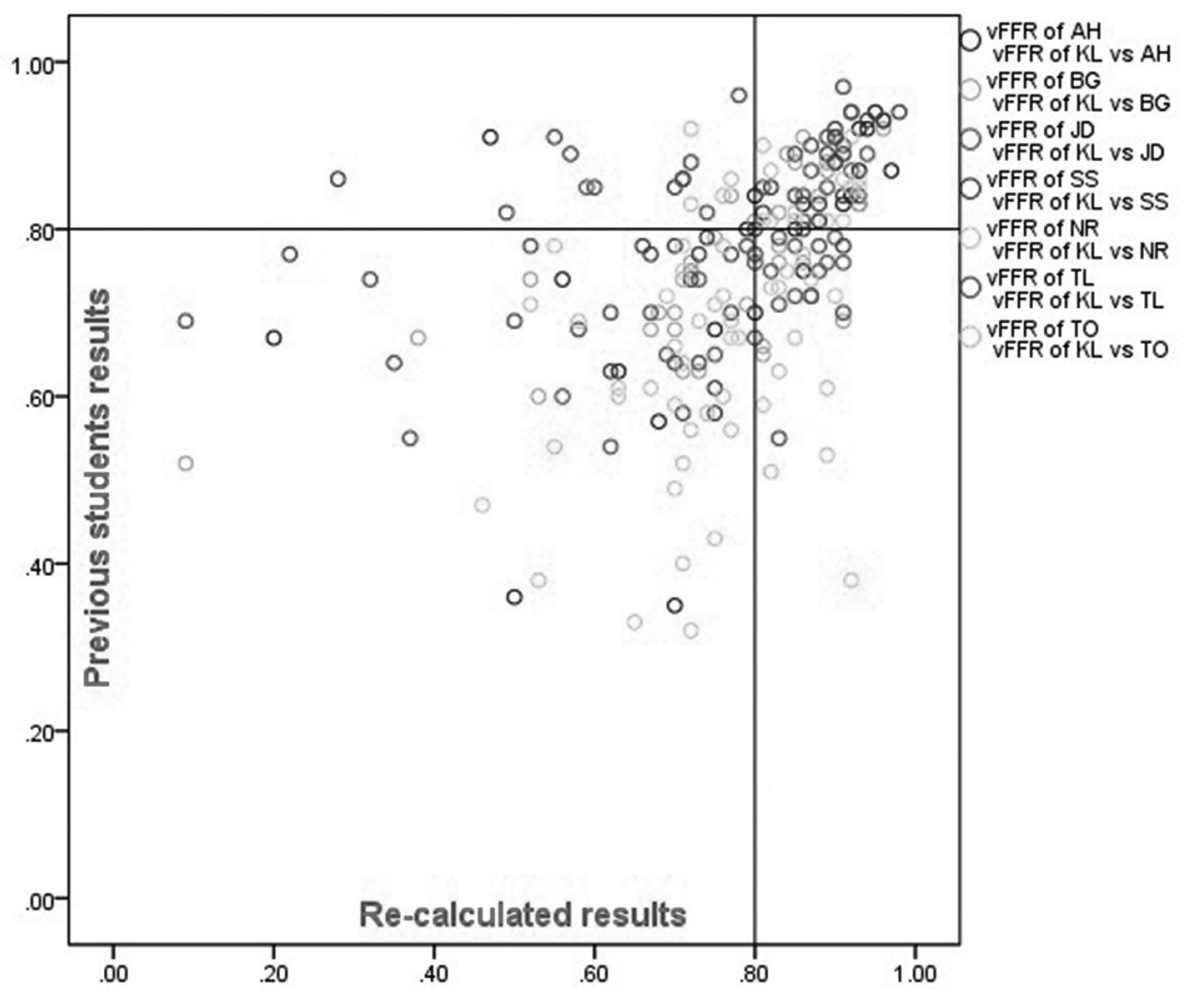

Abstract 17 Figure 2 Scatterplot of all previous students vFFR values compared to the 214 successfully re-calculated vFFR values

seven non-experts. The vFFRs were computed using the VIRTUheart $^{\mathrm{TM}}$ tool (University of Sheffield). Figure 1 shows an example from the workflow. The vFFR results of the expert and non-expert analysed were compared on the basis of the recommendation for percutaneous coronary intervention vs medical therapy and the reason for the differences were documented. Inter- and intra-expert differences and the impact of the expert decisions upon potential clinical management were also assessed.

Results The angiograms from 1098 patients with CAD were screened, from which 316 cases for vFFR analysis were identified as being suitable for processing. From these, one expert selected 264 consecutive cases for re-processing at random, of which 214 were successfully re-processed. Reasons for unsuccessful segmentation included inadequate images, poor opacification, overlap of vessels and unworkable geometry. The expert mean vFFR was 0.76 and the non-expert was 0.75 (mean per case difference 0.11 , SD 0.12 ), with $73 \%$ agreement and $27 \%$ disagreement about treatment strategy (see figure 2). Of those, $18 \%$ would have been incorrectly revascularised and 9\% incorrectly managed conservatively. The mean inter-observer (1st vs 2 nd expert) and intra-observer (1st vs 1 st expert) differences were 0.06 and 0.09 respectively, and agreement in management interpretations $89 \%$ and $90 \%$ respectively ( $\mathrm{p}<0.0001)$. The management interpretation, based upon expert vFFR analysis vs the original cardiologist's decision based upon the angiogram alone, revealed 37\% disagreement, with $23 \%$ incorrectly revascularised and $14 \%$ incorrectly managed conservatively.

Conclusion There is a large difference in vFFR modelling between expert and less expert modellers. The differences are due to errors in 3-D vessel construction. There is little interor intra-observer variation between expert modellers. However good the modelling system, training is required to produce accurate vFFR results. Expert vFFR can improve the clinical management of patients with $\mathrm{CAD}$, altering revascularisation decision in $37 \%$ cases.

Conflict of Interest None

\section{CLINICAL RISK MODEL TO PREDICT LIKELIHOOD OF HAVING GOOD LV FUNCTION POST MYOCARDIAL INFARCTION}

${ }^{1}$ David G Wilson*, ${ }^{1}$ Helen Routledge, ${ }^{2}$ Tom Johnson, ${ }^{3}$ Jessica Harris. ${ }^{1}$ Worcestershire Acute Hospitals NHS Trust; ${ }^{2}$ Bristol Heart Institute, Bristol Royal Infirmary; ${ }^{3}$ Clinical Trials and Evaluation Unit, Bristol Trials Centre, University of Bristol

\subsection{6/heartjnl-2019-BCS.17}

Background A national shortage of sonographers and NHS hospital beds challenges us in providing timely inpatient echocardiography to patients admitted to hospital with acute myocardial infarction (AMI). A clinical risk model to identify clinical predictors of having good LV function post AMI could potentially help risk-stratify patients for early discharge and expedited outpatient echocardiography.

Aim To develop a clinical risk score to predict the likelihood of having good LV function on transthoracic echocardiography post AMI.

Methods Data that had been collected for patients presenting to Worcestershire Acute Hospitals NHS Trust with AMI between July 2014 and November 2017 were used. These data had been collected as part of the Myocardial Infarction National Audit Project (MINAP). A clinical risk model was developed. Multiple imputation methods were used to deal with missing data. Logistic regression was used to determine to effect of these factors upon the outcome of good LV 